Supplemental Materials for the manuscript

\title{
Correlation of H Adsorption Energy and Nanoscale Elastic Surface Strain on Rutile $\mathrm{TiO}_{2}(110)$
}

Denis V. Potapenko, Gisele T. Gomes, and Richard M. Osgood, Jr.

Laboratory for Light-Surface Interactions, Department of Applied Physics and Applied Mathematics, Columbia University, New York, NY 10025, USA

S1. Observation of surface diffusion of $\mathrm{H}$ on $\mathrm{TiO}_{2}(110)$.

a)

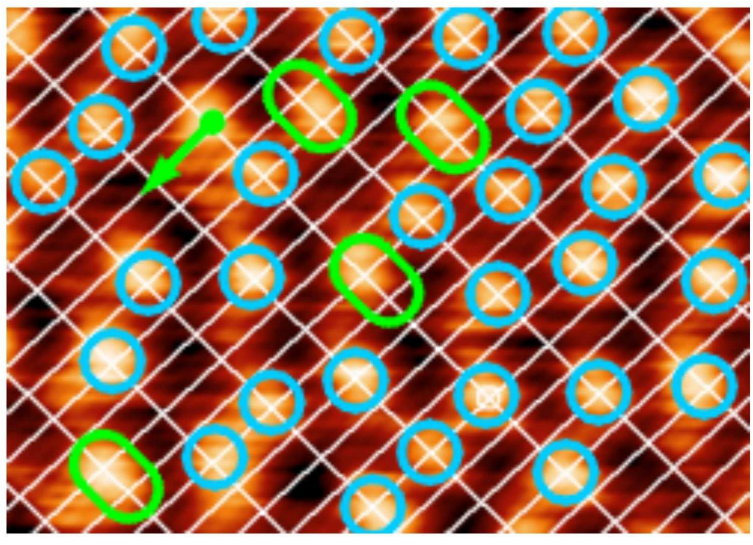

b)

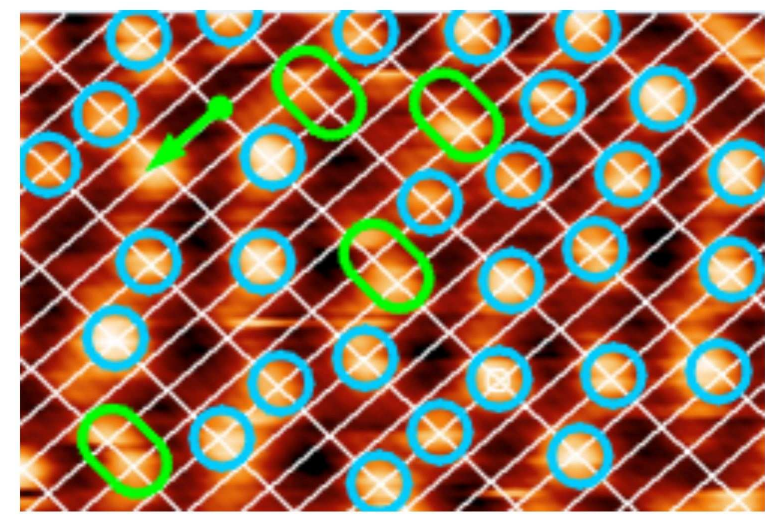

Figure S1. Two consecutive STM images of the same $6 \times 4 \mathrm{~nm}$ area of the $\operatorname{TiO}_{2}(110)$ surface taken 1 min apart. Bright features are the $\mathrm{OH}_{\mathrm{b}}$ groups. The cyan circles denote $\mathrm{OH}$ groups that did not move between the two scans, the green ovals show the $\mathrm{OH}$ features that underwent an along-the-row hopping event, and the green arrow show a 
hydrogen that has hopped across-the-row. The white overlaid grid marks unit cells of the (110) surface.

Figure S1 demonstrates that hydrogen atoms are mobile on $\mathrm{TiO}_{2}$ surface on the scale of minutes. Both along- and across-the-row diffusion mechanisms are active under the conditions of our experiments. Across-the-row diffusion at room temperature has been described by Wendt, et al. ${ }^{1}$ as assisted by water molecules rapidly diffusing along the Ti rows. However, note that the observation of along-the-row diffusion events at room temperature (RT) demonstrates that this type of diffusion is somewhat faster in our experiments than that in earlier results. ${ }^{2}$ We do not have a definitive explanation of this differences and only tentatively offer one explanation that the variation in diffusion rates may arise from different degree of sample surface reduction, since the initial BBOv concentration was $10 \%$ in our case and $7 \%$ in work by Li, et al. ${ }^{2}$

\section{S2. Calculation of the interaction potential from the radial distribution function.}

In order to estimate the pair-interaction potential $\mathrm{u}(\mathrm{r})$ between two isolated $\mathrm{OHb}$ groups from the experimentally determined radial distribution function $C\left(\mathrm{r}_{\mathrm{x}}, \mathrm{r}_{\mathrm{y}}=0\right)$ we have used the Ornstein-Zernike equation-based approach. ${ }^{3}$ In the manuscript we have justified the reduction of the problem to one-dimensional task. For this case we need to consider only one radial coordinate $r=\mathrm{r}_{\mathrm{x}}=0,1,2, \ldots$ Following the Ornstein-Zernike approach we introduce the total correlation function $h(r)=1-C\left(\mathrm{r}_{\mathrm{x}}, \mathrm{r}_{\mathrm{y}}=0\right) / \theta_{\mathrm{OH}}$. Then the direct correlation function $c(r)$, which represents the direct interaction between two $\mathrm{OH}_{\mathrm{b}}$ groups that are not mediated by other $\mathrm{OH}_{\mathrm{b}}$ groups, is defined by one-dimensional, discrete version of $\mathrm{O}-\mathrm{Z}$ equation:

$h(r)=c(r)+\theta \cdot \sum_{p=0}^{N} h(p) \cdot c(r-p)$

Here $\theta=\theta_{\mathrm{OH}}=0.20$. As $h(r)$ approached zero with increasing $r$, the summation was limited by $N=10$. The equation was solved with respect to $c(r)$ using Fourier transform. If we then denote the Fourier transforms of $h(r)$ and $c(r)$ as $H(k)$ and $C(k)$ respectively, then

$C(k)=\frac{H(k)}{1+\theta \cdot H(k)}$

The reverse Fourier transform of $C(k)$ then yields the $c(r)$ function. To find the pairinteraction potential from $h(r)$ and $c(r)$ we used the hypernetted-chain approximation ${ }^{3}$ that could be expressed as:

$u(r)=k T \cdot[\ln (h(r)+1)+c(r)-h(r)]$

All above calculations were performed using Microsoft Excel spreadsheet software. 
(1) Wendt, S.; Matthiesen, J.; Schaub, R.; Vestergaard, E. K.; Laegsgaard, E.; Besenbacher, F.; Hammer, B. Formation and Splitting of Paired Hydroxyl Groups on Reduced $\mathrm{TiO}_{2}$ (110). Phys. Rev. Lett. 2006, 96, 066107.

(2) Li, S. C.; Zhang, Z.; Sheppard, D.; Kay, B. D.; White, J. M.; Du, Y.; Lyubinetsky, I.; Henkelman, G.; Dohnalek, Z. Intrinsic Diffusion of Hydrogen on Rutile $\mathrm{TiO}_{2}(110)$. J. Am. Chem. Soc. 2008, 130, 9080-9088.

(3) McQuarrie, D. A. Statistical Mechanics; University Science Books: Sausalito, Calif., 2000. 